\title{
Outcome of osteochondral autograft transplantation for type- $V$ cystic osteochondral lesions of the talus
}

P. E. Scranton Jr, C. C. Frey, K. S. Feder

From University of Washington, Seattle, USA

P. E. Scranton Jr, MD, Orthopaedic Surgeon Orthopaedics International Ltd, 12333 NE 130th Lane, Suite 400, Kirkland, WA 98034, USA.

In C. C. Frey, MD, Orthopaedic Surgeon

K. S. Feder, MD, Orthopaedic Surgeon

1200 Rosecrans Ave, \#208 Manhatten Beach, California 90266-2470, USA.

Correspondence should be sent to Dr P. E. Scranton at $12333 \mathrm{NE}$ 130th Lane, Suite 400,

Kirkland,Washington 98034,

USA; e-mail:

piercescranton@hotmail.com

C2006 British Editorial Society of Bone and Joint Surgery doi:10.1302/0301-620X.88B5. $17306 \$ 2.00$

$J$ Bone Joint Surg [Br] 2006;88-B:614-19.

Received 13 October 2005 ;

Accepted after revision

27 January 2006

The treatment of osteochondral lesions of the talus has evolved with the development of improved imaging and arthroscopic techniques. However, the outcome of treatment for large cystic type- $\mathrm{V}$ lesions is poor, using conventional grafting, debridement or microfracture techniques.

This retrospective study examined the outcomes of 50 patients with a cystic talar defect who were treated with arthroscopically harvested, cored osteochondral graft taken from the ipsilateral knee.

Of the 50 patients, $45(90 \%)$ had a mean good to excellent score of $80.3(52$ to 90$)$ in the Karlsson-Peterson Ankle Score, at a mean follow-up of 36 months (24 to 83). A malleolar osteotomy for exposure was needed in $\mathbf{2 6}$ patients and there were no malleolar mal- or nonunions. One patient had symptoms at the donor site three months after surgery; these resolved after arthroscopic release of scar tissue.

This technique is demanding with or without a malleolar osteotomy, but if properly performed has a high likelihood of success.

The treatment of osteochondral lesions of the talus remains controversial, partly because of the advances in imaging, classification and the surgical techniques available. It is difficult to compare treatments of lesions that were poorly described before the advent of CT or MRI. There have been developments in arthroscopic imaging, instrumentation and non-invasive distraction techniques. ${ }^{1}$ Procedures involving drilling, microfracture, debridement, internal fixation and grafting have also evolved. There are few reports of the long-term clinical outcomes of specific forms of treatments for clearly defined lesions.

Various classifications and descriptions have been proposed since talar lesions were first described by König in $1888 .^{2}$ The lesions have been called osteochondritis dissecans, transchondral fractures, osteochondral fractures, dome fractures, flake fractures and chip fracture of the talus. ${ }^{3,4}$ With the advent of CT and MRI, further characteristics of these lesions have been described. ${ }^{5-7}$ There is now general agreement that this broad pathological spectrum of lesions should be called osteochondral lesions of the talus. ${ }^{1,8-11}$

Berndt and Harty ${ }^{4}$ first classified these lesions in 1959 as type-I to type-IV. It was a classification system based on both plain radiographs and the physical appearance of the talar sur- face at the time of surgery. However, with the development of CT and MRI, bone oedema and large unstable cystic defects were identified, for which various further descriptive classifications were proposed, ${ }^{12,13}$ and these have now been designated as type- $\mathrm{V} .{ }^{8,14}$

Drilling, debridement, microfracture, internal fixation or cancellous bone grafting have been described for the treatment of patients with osteochondral lesions of the talus. , $^{1,311,12,15,16}$ In several reports, when the patients with larger cystic lesions were evaluated separately, the results were poor. ${ }^{17,18}$ These larger lesions may be treated by osteochondral autograft transplantation. Good short-term clinical results have been published. ${ }^{8,19}$

The structure, stiffness, remodelling and splitline characteristics of osteochondral autografts have been studied in animal models, with encouraging results. ${ }^{20-22}$ However, there have been no long-term results for patients who have undergone an osteochondral autograft transplantation procedure for type-V lesions. We therefore report the long-term outcome in a group of patients for whom a type- $\mathrm{V}$ defect was replaced by an arthroscopically harvested osteochondral graft.

\section{Patients and Methods}

A retrospective review was undertaken of all patients undergoing an osteochondral auto- 
Scoring scale for assessment of functional results

Instability
None
1 or 2 times/year (during exercise)
1 or 2 times/month (during exercise)
Walking on uneven ground
Walking on even ground
Constant (severe), using ankle support
Pain
None
During exercise
Walking on uneven surface
Walking on even surface
Constant
Swelling
None
After exercise
Constant
Stiffness
None
Moderate (morning, after exercise)
Marked (constant, severe)
Work, sport activities and activities of daily living
Same as preinjury
Same work, less sports, normal leisure activities
Impaired working capacity, no sports, normal leisure activities
Severely impaired working capacity
Stair climbing
No problems
Impaired (instability)
Impossible
Running
No problems
Impaired
Impossible
Support
Ankle support during exercise
Ankle support during daily activities

Fig. 1

The Karlsson-Peterson Ankle Scoring method. ${ }^{23}$

graft transplantation procedure for a symptomatic type-V lesion of the talus in which autograft plugs were harvested arthroscopically from the ipsilateral knee. An Investigational Review Board approved this study and patient consent was obtained in all cases. Inclusion criteria were that the cystic lesion was between $8 \mathrm{~mm}$ and $20 \mathrm{~mm}$ in diameter. We excluded patients with larger lesions in which multiple allograft plugs or en bloc allografts were used. Most of these patients had also had previous surgical treatment, including drilling, fixation, cancellous grafting or debridement, and had also received at least six initial months of conservative treatment which included rest, immobilisation and physiotherapy before the index procedure. We recorded the following details: age, gender, duration of symptoms, a history of trauma, previous surgical treatment, location of the talar lesion (medial or lateral), and whether a medial malleolar osteotomy was necessary in the surgical approach. At follow-up, subsequent surgery (if any) was documented, as well as the presence or absence of knee pain. Follow-up radiographs at three months and one year were obtained either from our office or from the referring physicians. At final follow-up, the authors contacted each patient, who completed a Karlsson-Peterson Ankle Score questionnaire ${ }^{23}$ (Fig. 1). They were not asked to retrospectively assess what the Karlsson-Peterson score might have been pre-operatively, although each patient had presented with disabling symptoms of swelling, catching or pain on activity. They were asked if the surgery had improved their symptoms.

There were 53 patients ( 30 men, 23 women) identified who had this procedure between 1998 and 2003. One patient died of unrelated causes one year after the operation and two could not be traced. This left 50 patients in the study.

Operative technique. Our technique has been refined since it was described in $2001 .^{8}$ The site of the lesion determines whether a medial or a lateral approach is used. A posteromedial lesion usually requires a medial malleolar osteotomy. A lateral malleolar osteotomy has not so far been required, as adequate exposure is obtained with release of the anterior talofibular ligament, anterior subluxation and forced plantar flexion. Anteromedial or lateral lesions require only an arthrotomy.

The anaesthetised patient is placed in the supine position. For a posteromedial lesion, a curved longitudinal incision is made over the medial malleolus, sufficient to expose the anteromedial and posteromedial ankle joint. The saphenous vein, neurovascular and tendon structures are protected. An anteromedial ankle arthrotomy is carried out and the joint inspected. Impingement spurs or loose bodies are removed. If the lesion can be clearly visualised and accessed by plantar flexion, a malleolar osteotomy is not necessary. If not, a malleolar osteotomy is performed.

The medial malleolus is predrilled with two $0.53 \mathrm{~mm}$ pins and over-drilled with an AO Synthes $2.7 \mathrm{~mm}$ cannulated drill (Synthes GmbH, Solothurn, Switzerland), to a depth that crosses into the tibial plafond. The holes should diverge slightly. A marker pen is used to draw a $45^{\circ}$ osteotomy cut from the superior medial malleolus, coming down to the junction of the tibial plafond and medial colliculus (Fig. 2). This creates a plane for an oscillating saw. The last one-eighth is completed using an osteotome. The assistant distracts the ankle laterally at the time of osteotomy, ensuring protection of the talar articular surface. The osteotomised medial malleolus is retracted inferiorly to expose the talus.

The damaged surface of the talus is debrided. By correlating the CT or MR images with intra-operative findings, the centre of the cystic lesion is determined and is drilled perpendicularly (Fig. 3). If the lesion is on the dome, it is 


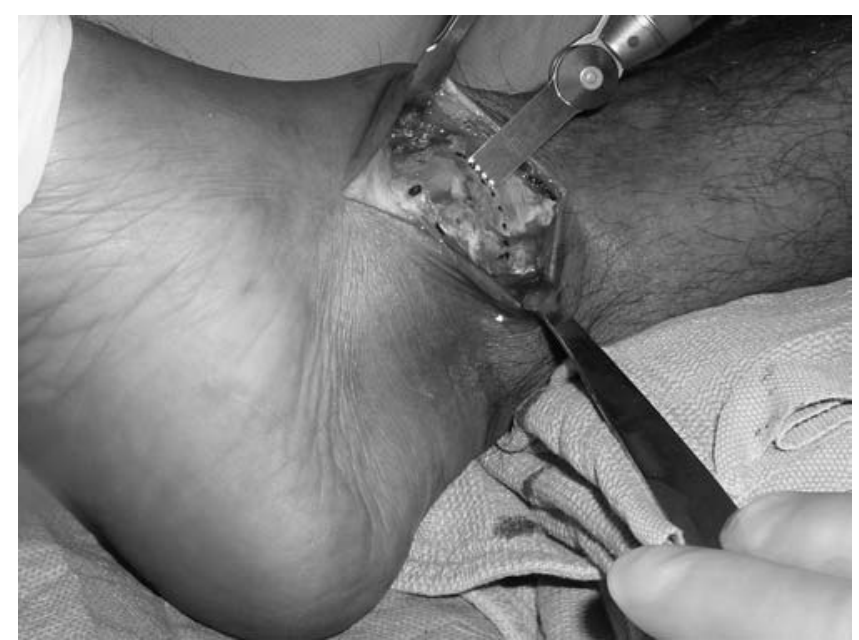

Fig. 2

An intra-operative photograph of an exposed right medial malleolus. Two malleolar holes have been pre-drilled. The Homan retractors protect neurovascular and tendinous structures. The plane of the osteotomy has been marked.

drilled straight down from the top. If it is on the shoulder of the talus it is drilled at a $45^{\circ}$ angle. The size of the lesion can be determined both from direct measurement and from the CT or MRI scans. In either case, the pin is advanced to a depth of about $15 \mathrm{~mm}$ and is over-drilled to a depth of at least $12 \mathrm{~mm}$. Some of the debris is lavaged and some will be forced into the interstices of the cyst. The pin is removed and the depth measured with a sizing rod. If necessary, a second or even a third core can be created adjacent to the first where 'nesting' of grafts is necessary. Two techniques are used, depending on the shape of the cyst. An oblong cyst allows two grafts to be nested side by side. In a larger (i.e. $\leq 20 \mathrm{~mm}$ ) cyst it is possible to nest three $7 \mathrm{~mm}$ to $8 \mathrm{~mm}$ grafts, filling in the interstices with a small amount of cancellous graft. Attention is then turned to the knee.

The arthroscope is introduced through an anterolateral portal and a routine inspection of the knee joint carried out. The appropriate size of donor tube is introduced with the obturator slightly protruding. This allows the tube to slip easily through the medial portal and clear the fat pad without catching on synovium. If the talar graft to be matched is from the flatter dome region, the donor tube is positioned more vertically over the flatter sulcus terminalis portion of the notch. The knee is partially extended to accommodate this vertical angle. If the graft requires a more curved articular surface to fit a lesion on the corner of the talus, the donor tube is positioned on the edge of the lateral notch of the femoral trochlea. In each instance, the obturator is then allowed to slide back and the donor tube driven into a depth of $15 \mathrm{~mm}$. It is twisted $90^{\circ}$ clockwise under pressure, back again, and then a full clockwise revolution. The tube and graft are withdrawn. Back-filling of the defect is not necessary.



Fig. 3

The medial malleolus has been retracted inferiorly. A pin has been drilled perpendicularly into the centre of the defect.

The cored osteochondral graft is measured. If a full 15 $\mathrm{mm}$ has been harvested, it is carefully rongeured back to a tapered $12 \mathrm{~mm}$ to match the recipient hole in the talus. It is then introduced into the talar hole in optimal orientation for articular congruity and until it is flush with the articular surface. The medial malleolus is replaced and fixed with two $40 \mathrm{~mm}$ cannulated $4.0 \mathrm{~mm}$-diameter cancellous AO screws. The slight diversion of the screws ensures that the malleolus will not migrate superiorly.

The patient remains non-weight-bearing in a bootwalker for three weeks, non-weight-bearing but out of the boot walker for the next three weeks and then weight-bearing in the bootwalker for the final three weeks, followed by routine physiotherapy.

For anteromedial, anterolateral and posterolateral talar lesions, the harvesting technique is the same, but without a malleolar osteotomy.

\section{Results}

All 50 patients were evaluated and at final follow-up filled out the Karlsson-Peterson Ankle Score questionnaire. Their mean age was 36 years (17 to 56) and all had lesions ranging from $8 \mathrm{~mm}$ to $20 \mathrm{~mm}$ in diameter, confirmed by CT, MRI, or both. The mean follow-up was 36 months (24 to 83). All patients had standing anteroposterior and lateral radiographs of the ankle at six weeks and one year postoperatively. Because of the expense, MRI scans were not used for routine post-operative evaluation in patients who enjoyed full activity with no, or only minimal, symptoms. If there was a new injury or persistent symptoms, an MRI scan was obtained.

The duration of symptoms was difficult to ascertain, owing to the delay in the development of further symptoms 
after the initial traumatic event. The duration was therefore divided into either more than or less than one year. A total of 40 patients had symptoms for more than one year before the diagnosis of an osteochondral lesion of the talus was made and ten had symptoms for less than one year. Before the onset of symptoms, 42 patients ( $84 \%$ ) could remember a traumatic event, either a sprain or a fracture of the ankle.

Of the 32 patients $(64 \%)$ who had undergone one or more operations on the ankle previously, including arthroscopic or open debridement, curettage, drilling, internal fixation or grafting, $11(22 \%)$ had two or more previous operations.

A total of 45 patients $(90 \%)$ were satisfied with the outcome and had a mean ankle score according to the Karlsson-Peterson questionnaire of 80.3 (52 to 90). Their mean score for ankle stiffness was 3.2/5.0 (2 to 5). Intermittent aching or pain because of sports or work resulted in a lower mean pain score of $14.2 / 20$ (0 to 20 ). Three patients graded the outcome as fair, with scores of 42,42 and 49 .

A malleolar osteotomy was carried out in 26 patients $(52 \%)$. There were ten lateral and 14 medial lesions, which did not require an osteotomy. There were no cases of malunion or nonunion of the malleolar osteotomy.

Further surgery was required in 17 patients (34\%) and ten $(20 \%)$ had an arthroscopy and debridement. Malleolar screws were removed in four patients $(8 \%)$, while there were two $(4 \%)$ with severe degenerative changes who had a further attempted osteochondral autograft transplantation procedure and a subsequent arthrodesis of the ankle. One had symptomatic scar tissue debrided from the knee. The mean Karlsson-Peterson Ankle Score at final follow-up for all 50 patients was 76.2 (5 to 100$)$.

\section{Discussion}

The standardisation of nomenclature and classification of osteochondral lesions of the talus has improved our understanding of the outcome following treatment. Most descriptive terms do not cover the complete spectrum of articular and bony pathology seen in these lesions of the talus. The arthroscopic classification proposed by Pritsch et $\mathrm{al}^{12}$ does not take into consideration the underlying subchondral bony defect in the talus. The same is true for descriptive MRI classifications. ${ }^{6,7,13}$ The articular defect may not be identified on MRI. Characterising these large cystic defects as type-V lesions keeps the application of treatment options within the algorithm previously established by Berndt and Harty. ${ }^{4}$ In type-V lesions, the cystic defect must be filled and articular surface congruency restored and stabilised.

The majority of our patients had symptoms for more than one year before the diagnosis was made. This diagnosis was confirmed in all cases by CT or MRI, often after several series of normal radiographs of the ankle. Verhagen et $\mathrm{al}^{24}$ found that CT or MRI was most reliable in diagnosing osteochondral lesions of the talus. Physical examination may be normal. Symptoms of synovitis, effusion, or painful catching or snapping can be intermittent or absent. A history of previous trauma and any of these other symptoms, should alert the examiner to the possibility of an osteochondral lesion of the talus. ${ }^{3,4}$

This outcome study was confined to 50 patients with type- $\mathrm{V}$ lesions treated by an osteochondral autograft from the ipsilateral knee. Only one patient had symptoms of painful scar tissue requiring arthroscopic debridement of the knee. Except for early pain and an effusion, the rest had no knee symptoms after three months. The methods of treatment which have been used for patients with osteochondral lesions of the talus include rest, immobilisation, casting, non-steroidal anti-inflammatory medication, protected movement or crutches, retro- and antegrade drilling, curettage, debridement, retro- and antegrade grafting, metallic or biodegradable screw or peg fixation, allografting, autografting, mosaicplasty, autograft Carticel (Genzyme, Cambridge, Massachusetts) procedures, autograft Carticel sandwich procedures and tissue-engineered cartilage grafting. ${ }^{4,11,12,15-19,25-27}$ Many of these are quite successful, but larger cystic lesions have not done well with the more conservative surgical alternatives.

The main drawback of our study is that it is retrospective. When we began this type of surgery, the patients presenting had been referred for an ankle arthrodesis. We offered the osteochondral autograft transplantation procedure in an attempt to salvage ankle function. The historical controls of Robinson et $\mathrm{al}^{17}$ and Kolker et $\mathrm{al}^{18}$ give perspective to alternative forms of treatment in patients with large cystic lesions. Robinson et $\mathrm{al}^{17}$ found that drilling and debridement had a poor outcome in $53 \%$ of patients with large cystic lesions. Kolker et $\mathrm{al}^{18}$ found that only $46.2 \%$ of patients who had undergone antegrade cancellous autografting were satisfied with the outcome. They recommended that grafting should be used 'with extreme caution'. Failed previous treatment was common in our series, with 32 patients $(64 \%)$ having had at least one previous operation.

Our surgical technique includes two important modifications. First, the cyst is drilled rather than cored. Many talar cysts are irregular in shape. As the surgeon drills into them, underlying vascular bone creates cancellous debris which can line the hole, in effect as a compressed graft slurry. This drilling allows for the creation of a more cylindrical recipient hole for the donor graft. It has allowed us to treat larger cysts using the $10 \mathrm{~mm}$ osteochondral autograft transplantation system, inserting only one plug. Two or three plugs can be 'nested'.

Secondly, the donor plugs are inserted under direct vision, instead of extruding blindly from the donor tube into the recipient hole. This modification is of paramount importance. Discrepancies in the depth or shape of the graft as it sits in the talus will spell the difference between success and failure. Huang et $\mathrm{al}^{20}$ showed that small incongruities can remodel, provided they do not exceed $1 \mathrm{~mm}$ in either direction. Nam et $\mathrm{al}^{21}$ showed that the osteochondral graft 


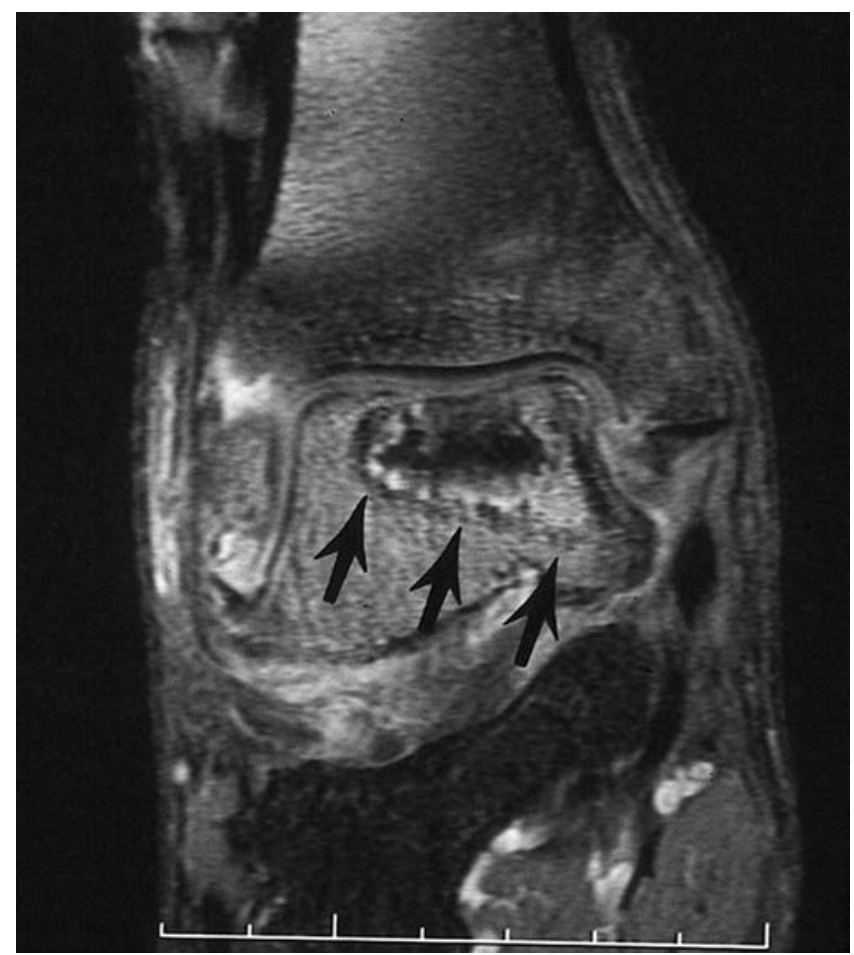

Fig. 4

Coronal MRI scan of a 29-year-old patient with a 20-mm diameter osteochondral lesion of the talus one year after being treated for a Weber-B fracture of the ankle. There is cyst formation after failed retrograde bone grafting.

will remodel its stiffness characteristics in order to mimic the surrounding cartilage. The split-line pattern or tidemark discrepancy of the host versus graft does not appear to matter as long as the graft is within $1 \mathrm{~mm}$ of congruity to the host tissue. Therefore, a precise orientation of the graft and line-to-line height match is critical.

We used the Karlsson-Peterson Ankle Scoring System at final follow-up to evaluate outcome. This system measures pain, swelling, stability, work, stair-climbing, running, and whether or not an ankle support is necessary.

Alternative treatments for large type- $\mathrm{V}$ lesions include the mosaicplasty and the Carticel techniques..25,27 Good results have been reported with both. Hangody et $\mathrm{al}^{27}$ described good to excellent results in 34 of 36 cases, with a two- to seven-year follow-up. His technique involved an open knee arthrotomy for graft harvesting and the nesting of multiple $4.5 \mathrm{~mm}$ or $6.5 \mathrm{~mm}$ diameter grafts. His results appeared to validate the technique. However, the nesting of multiple grafts was technically more difficult than a single graft and the arthrotomy of the knee caused symptoms for up to one year in $17 \%$ of patients. There were also multiple cartilage gaps in the circular nested grafts. It would take three $4.5 \mathrm{~mm}$ grafts to fill the defect of a single $10 \mathrm{~mm}$ osteochondral autograft transplantation graft. Also, as shown by Huntley et $\mathrm{al}^{28}$ a periphery of chondrocytes around each core dies after mosaicplasty harvest. They esti-



Fig. 5a

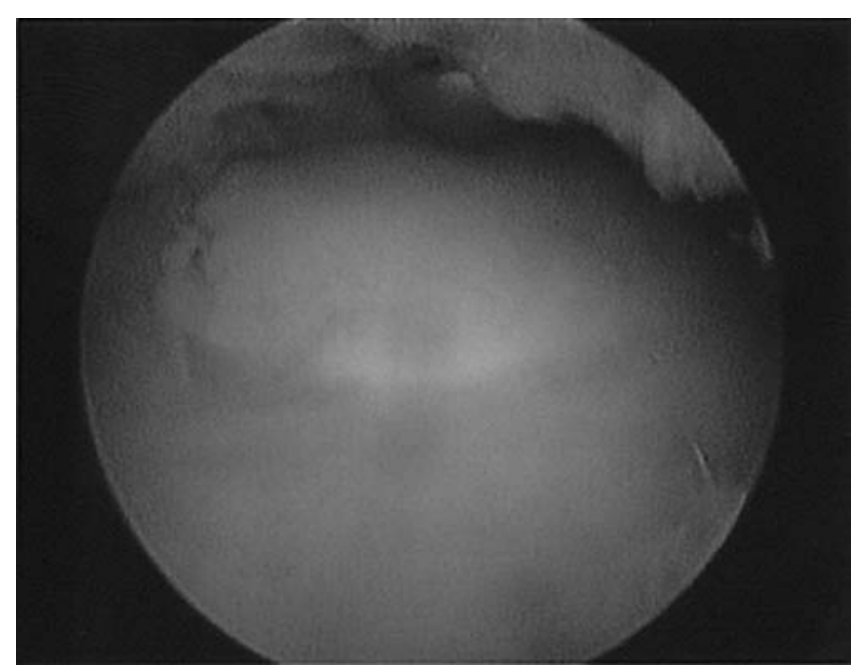

Fig. $5 b$

Figure 5a-Radiograph of a 16-year-old patient one year after failed internal fixation of a type-IV lesion. The talar fragment was avascular. Figure $5 b$ - The appearance of the articular surface of the talar graft, of the patient in Figure $5 \mathrm{a}$, after removal of the snapping scar tissue.

mated that $24 \%$ of the chondrocytes implanted from the graft harvester were dead. It seems simpler to insert one larger, arthroscopically harvested graft.

The Carticel procedure introduced by Peterson has enjoyed great success in the knee joint. ${ }^{25}$ It has more recently been applied to the ankle joint without, however, any long-term follow-up reports. The technique is expen- 
sive, demanding, and involves a three-month wait for cultured chondrocytes. Horas et $\mathrm{al}^{29}$ showed that subjective improvement in autograft Corticel patients was slower than for osteochondral autograft transplantation patients. Further second-look biopsies showed fibrocartilage only in the autograft Carticel patients, compared with hyaline cartilage in the osteochondral autograft transplantation patients. Unless there is broad destruction of the talar articular surface, we believe the osteochondral autograft transplantation procedure to be simpler and cheaper. Furthermore, a patient treated with the osteochondral autograft transplantation technique will be healed and well into physical rehabilitation by the time a Carticel patient is receiving their cultured chondrocyte graft.

There were 15 minor secondary procedures for medial malleolar screw removal or arthroscopic debridement. Three patients reported a fair result, subjectively improved but not satisfied, while two with extensive associated degenerative arthritis eventually underwent arthrodesis. Figure 4 illustrates an extreme case of a focal lesion with avascular collapse after open reduction and internal fixation of a Weber-B ankle fracture ${ }^{30}$ and failed retrograde graft. This patient had been advised to have an ankle arthrodesis. He underwent a malleolar osteotomy with two 7 $\mathrm{mm}$ grafts and a $5 \mathrm{~mm}$ graft nested into the defect. It is five years since the operation and he has enjoyed four more years in the national football league. Figure 5 illustrates a patient with failed internal fixation of a Berndt and Harty type-IV lesion, who was referred for an arthrodesis. An arthroscopic second look for scar debridement two years post-operatively showed an underlying excellent appearance of the talar graft. Patients with type-V lesions who undergo this procedure should be advised that they may develop later catching of scar tissue or pain at a screw head requiring a further simple procedure.

\section{Supplementary Material}

$\ddot{e}$ A further opinion by Mr Andrew Robinson is available with the electronic version of this article on our website at www.jbjs.org.uk

No benefits in any form have been received or will be received from a commercial party related directly or indirectly to the subject of this article.

\section{References}

1. Ferkel RD. Arthroscopic treatment of osteochondral lesions, soft-tissue impingement and loose bodies. In: Pfeffer GB, ed. Chronic ankle pain in the athlete. Rosemont: AAOS 2000:43-70.

2. Konig F. Uber freie koper in den gelenken. Dtsch Z Chir 1888;27:90-109.

3. Flick AB, Gould N. Osteochondritis dissecans of the talus (transchondral fractures of the talus): review of the literature and new surgical approach for medial dome lesions. Foot Ankle Int 1985;5:165-85.
4. Berndt AL, Harty M. Transchondral fractures (osteochondritis dissecans) of the talus. J Bone Joint Surg [Am] 1959;41-A:988-1020.

5. Zinman C, Reis ND. Osteochondritis dissecans of the talus: use of the high resolution computed tomography scanner. Acta Orthop Scand 1982;53:697-700.

6. De Smet AA, Fisher DR, Burnstein MI, Graf BK, Lange RH. Value of MR imaging in staging osteochondral lesions of the talus (osteochondritis dissecans): results in 14 patients. Am J Roentgenol 1990;154:555-8.

7. Dipaola JD, Nelson DW, Colville MR. Characterizing osteochondral lesions by magnetic resonance imaging. Arthroscopy 1991;7:101-4.

8. Scranton PE Jr, McDermott JE. Type V osteochondral lesions of the talus with ipsilateral knee osteochondral autografts. Foot Ankle Int 2001;22:380-4.

9. Thompson JP, Loomer RL. Osteochondral lesions of the talus in a sports medicine clinic: a new radiographic technique and surgical approach. Am J Sports Med 1984; 12:460-3.

10. Canale ST, Belding RH. Osteochondral lesions of the talus. J Bone Joint Surg [Am] 1980;62-A:97-102.

11. Parisien JS. Arthroscopic treatment of osteochondral lesions of the talus. Am J Sports Med 1986;14:211-17.

12. Pritsch M, Horoshovski H, Farine I. Arthroscopic treatment of osteochondral lesions of the talus. J Bone Joint Surg [Am] 1986;68-A:862-5

13. Ferkel RD. Articular surface defects, loose bodies, and osteophytes: osteochondral lesions of the talus. In: Whipple TL, ed. Arthroscopic surgery: the foot and the ankle. Philadelphia: Lippincott-Raven, 1996:145-84.

14. Hepple S, Winson IG, Glew D. Osteochondral lesions of the talus: a revised classification. Foot Ankle Int 1999;20:789-93.

15. Angermann P, Jensen P. Osteochondritis dissecans of the talus: long-term results of surgical treatment. Foot Ankle Int 1989;10:161-3.

16. Kumai T, Takahura Y, Higashiyama I, Tamai S. Arthroscopic drilling for the treatment of osteochondral lesions of the talus. J Bone Joint Surg [Am] 1999;81-A 1229-35.

17. Robinson DE, Winson IG, Harries WJ, Kelly AJ. Arthroscopic treatment of osteochondral lesions of the talus. J Bone Joint Surg [Br] 2003;85-B:989-93.

18. Kolker D, Murray M, Wilson M. Osteochondral defects of the talus treated with autologous bone grafting. J Bone Joint Surg [Br] 2004;86-B:521-6.

19. Al-Shaikh RA, Chou LB, Mann JA, Dreben SM, Prieskorn D. Autologous osteochondral grafting for talar cartilage defects. Foot Ankle Int 2002;23:381-9.

20. Huang FS, Simonian PT, Norman AG, Clark JM. Effects of small incongruities in a sheep model of osteochondral autografting. Am J Sports Med 2004;32:1842-8.

21. Nam EK, Makhsous $\mathbf{M}$, Koh J, et al. Biomechanical and histological evaluation of osteochondral transplantation in a rabbit model. Am J Sports Med 2004;32:308-16.

22. Koh JL, Wirsing K, Lautenschlager E, Zhang LO. The effect of graft height mismatch on contact pressure following osteochondral grafting: a biomechanical study. Am J Sports Med 2004;32:317-20.

23. Karlsson J, Peterson L. Evaluation of ankle joint function: the use of a scoring scale. The Foot 1991;1:15-19.

24. Verhagen RA, Mass M, Dijkgraaf MG, et al. Prospective study on diagnostic strategies in osteochondral lesions of the talus: is MRI superior to helical CT? J Bone Joint Surg $[B r] 2005 ; 87-B: 41-6$

25. Brittberg M, Lindahl A, Nilsson A, et al. Treatment of deep cartilage defects in the knee with autologous chondrocyte transplantation. Engl J Med 1994;331:889-95.

26. Agung M, Ochi M, Adachi N, et al. Osteochondritis dissecans of the talus treated by the transplantation of tissue-engineered cartilage. Arthroscopy 2004;20:1075-80.

27. Hangody L, Kish G, Módis L, et al. Mosaicplasty for the treatment of osteochondritis dissecans of the talus: two to seven year results in 36 patients. Foot Ankle Int 2001:22:552-8.

28. Huntley JS, Bush PG, McBirnie JM, Simpson AH, Hall AC. Chondrocyte death associated with human femoral osteochondral harvest as performed for mosaicplasty. J Bone Joint Surg [Am] 2005;87-A:351-60.

29. Horas U, Pelinkovic D, Herr G, Aigner T, Schnettler R. Autologous chondrocyte implantation and osteochondral cylinder transplantation in cartilage repair of the knee joint: a prospective, comparative trial. J Bone Joint Surg [Am] 2003;85-A 185-92.

30. Hamilton WC. Traumatic disorders of the ankle. New York: Springer-Verlag, 1984 